\title{
Variabilidad de cepas de Hirsutella thompsonii, a partir de ácaros fitófagos en tres sistemas terrestres del estado de Colima, México
}

\author{
Variability of Hirsutella thompsonii strains, isolated from phytophagous mites from three terrestrial \\ systems in the State of Colima, México
}

\author{
José Luis Rosas-Acevedo ${ }^{1 *}$ y Laura Sampedro-Rosas ${ }^{1}$ \\ ${ }^{1}$ Unidad de Ciencias de Desarrollo Regional. Universidad Autónoma de Guerrero. Calle Pino s/n Col. El Roble, 39640 Acapulco, Guerrero, México. \\ *Correspondencia: jlrosas71@hotmail.com
}

\begin{abstract}
Resumen. Entre 1999 y 2004 se obtuvieron diez cepas del hongo Hirsutella thompsonii Fisher, a partir de ácaros infectados, en 9 localidades de 3 de los 11 sistemas terrestres que tiene el estado de Colima, México: Llanura Costera de Tecomán, Llanura Costera de Cuyutlán y Valle del río Armería. De las 10 cepas, 5 pertenecen a la variedad thompsonii que es de crecimiento micelial mullido, ligeramente elevado, de color gris a gris verdoso, y 5 a la variedad sinematosa Samson, McCoy y O’Donnell, de apariencia plana y coloración blanco a amarillo. Los hospedantes fueron araña roja (Tetranychus urticae Koch), eriófidos (Aceria guerreronis Keifer y Phyllocoptruta oleivora Ashmead) y brevipálpidos (Brevipalpus phoenicis Geijkes) sobre cítricos como hospedero de 8 de los 10 aislamientos. De ellos, sólo la cepa HtM130 de H. thompsonii var. thompsonii presentó formación de exudados en la fase esporulativa, característica que le da mayor potencial de manejo en estrategias para el control de ácaros fitopatógenos. El tipo de crecimiento y la coloración de las cepas no se correlacionan con el sistema terrestre donde se encontraron, ni con las condiciones climáticas imperantes en ellos. La presente contribución demuestra la importancia de valorar la presencia de enemigos naturales autóctonos para garantizar un mejor establecimiento bajo las condiciones agroclimáticas de la región donde se pretenda utilizarlos, y antes de introducir microorganismos comerciales o aislamientos de otros sitios para no afectar los sistemas naturales de regulación.
\end{abstract}

Palabras clave: biodiversidad, entomopatógeno, enemigos naturales, hongo autóctono.

\begin{abstract}
Between 1999 and 2004, ten strains of the fungus Hirsutella thompsonii Fisher were isolated from infected mites, in 9 localities of 3 of the 11 terrestrial systems found in the state of Colima, Mexico: coastal plain of Tecomán, coastal plain of Cuyutlán, and the valley of the Armería river. Of the 10 strains, 5 belong to var. thompsonii, whose growth is fluffed mycelial, slightly elevated, gray to greenish gray, and 5 to var. sinematosa, with a flat appearance, white to yellow. The hosts were two-spotted spider mite (Tetranichus urticae), Eriophidae (Aceria guerreronis and Phyllocoptruta oleivora) and Brevipalpidae (Brevipalpus phoenicis), Citrus spp. being the host of the mites in 8 of the 10 isolations. Of the 10 strains, only one HtM130 strain of $H$. thompsonii var. thompsonii displayed exudate formation in the sporulative phase, characteristic that gives greater potential for implementation in management strategies for the control of phytophagous mites. Strain coloration and growth are not correlated with the terrestrial system where they were found, nor to prevailing climatic conditions. This contribution demonstrates the importance of assessing the presence of native natural enemies, before introducing commercial microorganisms or isolations from elsewhere so as not to affect the natural regulatory systems.
\end{abstract}

Key words: biodiversity, entomopathogen, natural enemies, native fungus.

\section{Introducción}

Extensas revisiones sobre los enemigos naturales de los ácaros citan los hongos Entomophtorales y Deuteromicetos como los principales grupos de patógenos para estos artrópodos (Chandler et al., 2000; Van Der Geest et al., 2000). Estos entomopatógenos se encuentran en la naturaleza y con frecuencia causan importantes descensos en

Recibido: 26 enero 2005; aceptado: 24 octubre 2005 las poblaciones de los ácaros y son los factores ambientales (temperatura, humedad y la radiación solar), los que afectan directa o indirectamente tanto a su persistencia como a su sobrevivencia (Roberts y Campbell, 1977). Para compensar la mortalidad natural y maximizar su sobrevivencia en el ambiente, muchas especies de hongos producen elevadas cantidades de esporas, clamidosporas y otras estructuras de resistencia, exudados y toxinas (Mazet y Vey, 1995; Boucias and Pendland, 1998; Rosas et al., 2003).

El género Hirsutella Patouillard, pertenece al phyllum 
Deuteromycota (Fungi Imperfecti), clase Hyphomycetes y orden Moniliales; éste se ha relacionado con los géneros Cordyceps e Hypocrella (Orden Sphaeriales, Clase Pyrenomycetes,) del Phyllum Ascomycota (Mains, 1951; Hodge et al., 1997). Spears and Yothers (1924) lo encontraron parasitando a la negrilla de la naranja Phyllocoptrupta oleivora Ashmead en la Florida; 25 años más tarde, Fisher et al. (1949) citaron la epizootia de Hirsutella thompsonii sobre P.oleivora, la cual provocaba que los eriófidos se tornaran lentos en sus movimientos y cambiaran de coloración (Muma, 1958).

Se citan parasitando de forma natural a eriófidos y tetraníquidos cinco especies de Hirsutella: H. nodulosa Petch., H. kirchneri (O. Rostr.) Minter, Brady y Hall, $H$. necatrix Minter, Brady y Hall, $H$. gregis Minter, Brady y Hall y H. thompsonii (Cabrera y Domínguez, 1987; McCoy et al. 1988; McCoy, 1996; Rosas y Sampedro, 1992). Cada una tiene características especiales; particularmente, $H$. thompsonii está considerada una especie pleomórfica, por lo que se ha separado en 3 grupos morfológicos distintos que son las variedades: sinematosa Samson, McCoy y O'Donnell, vinacea Samson, McCoy y O'Donnell y thompsonii (Samson, et al., 1980).

El estado de Colima se localiza a $450 \mathrm{~km}$ al oeste de la ciudad de México, presenta dos zonas climático-geográficas: templado y trópico seco (García, 1987). Las temperaturas promedio de noviembre a abril son de 23 a $27^{\circ} \mathrm{C}$. Una de las principales actividades económicas es la agrícola (cítricos y otros frutales), donde los ácaros (Eriophyidae y Tetranychidae) son importantes plagas que se combaten con el uso constante de productos químicos, lo que los hace adquirir rápida resistencia como método de supervivencia ante el frecuente contacto con los insumos químicos (Tsagkarakou et al., 1996; Navajas et al., 1998; Wilson et al.1999).

Es importante aislar los enemigos autóctonos en cada región y conocer la biodiversidad existente para establecer alternativas de control y mitigar el impacto que los productos químicos pueden producir al ambiente y a la salud. En la zona citrícola del estado de Colima, aún no se explota la potencialidad del hongo $H$. thompsonii para la regulación natural de T. urticae y P. oleivora, principales ácaros fitófagos del oro verde colimense, en tanto que los productos químicos como medio de control se siguen utilizando en exceso.

\section{Materiales y métodos}

Área de estudio. El estado de Colima se divide en 11 sistemas con 34 paisajes terrestres, según el Ordenamiento Ecológico del territorio del estado de Colima (Dirección de Ecología de Colima,1993). De 1999 a 2004 se visitaron localidades de los 11 sistemas terrestres (Cuadro 1), cercanos a agroecosistemas citrícolas y otros cultivos (mangos, guayaba, tamarindo, café, caña, etc.), además se inspeccionó la flora silvestre aledaña, con problemas de ácaros fitofágos, especialmente tetraníquidos y eriófidos. Colecta de plantas con ácaros infectados. Para la localización de los ácaros, se seleccionó al azar una planta en cada uno de los cultivares visitados o áreas aledañas a ellos. La colecta se realizó por inspección visual (lupa de bolsillo 4X) al follaje afectado por ácaros, para detectar especímenes infectados por el hongo, los cuales se colocaron sobre tiras de papel absorbente (periódico) y se introdujeron en bolsas de plástico para su transporte e inspección en el laboratorio (Rosas y Sampedro, 1991), las hojas se examinaron al microscopio esteroscópico (50X) para la observación y el montaje de aquellos especímenes muertos con características de estar afectados por alguna enfermedad fungosa, los cuales se colocaron con ayuda de una aguja fina de siembra (00) en portaobjetos con lactofenol y se examinaron al microscopio (640X) para identificar si estaban afectados por H.thompsonii.

Aislamiento. El hongo Hirsutella spp., como todos los hifomicetos entomopatógenos, con frecuencia puede ser aislado de cadáveres de insectos (Lacey, 1997) aunque, para tener éxito en el aislamiento de $H$. thompsonii parasitando ácaros, no se debe desinfectar la superficie de los cadáveres (Rosas y Sampedro, 1991). Para el aislamiento, se usó el medio selectivo McCoy "E", desarrollado para el aislamiento de $H$. thompsonii (McCoy and Kanavel, 1969), modificado con la adición de malta al que se llamó MEA (Rosas et al., 2003). Se usaron placas con este medio, incubadas por 2 días a $27^{\circ} \mathrm{C}$ fueron usadas como substrato para los aislamientos. Bajo microscopio estereoscópico, se observaron las hojas o frutos colectados en localidades de los 11 sistemas terrestres en que se divide el estado de Colima. Se tomaron los ácaros infectados, con ayuda de una aguja estéril número 00, colocando hasta siete ácaros sobre cada placa con MEA, haciendo una punción sobre el medio de cultivo. Posteriormente se incubaron por 7 días, realizando observaciones diarias los 3 primeros días para eliminar contaminantes, como bacterias u otros hongos de desarrollo rápido, alrededor de los puntos de siembra, que pueden enmascarar o dificultar el crecimiento del entomopatógeno y su aislamiento (Rosas y Sampedro, 1991). Pasados los 7 días de incubación, las colonias compactas de crecimiento lento (característico de $H$. thompsonii) fueron trasferidas a tubos Eppendorf de $1.5 \mathrm{ml}$ con medio MEA para su conservación y corroborar la identificación. Identificación del hongo. Se prepararon microcultivos de cada aislamiento para estudiar la morfología del micelio, fiálides y conidios característicos de $H$. thompsonii, utilizando las claves de Samsom et al. (1980) y Rombach y Winkelhoff (1981). Según Minter y Bradi (1980) y Roberts (1989), el hongo se ubica dentro de la siguiente clasificación:

División Amastigomycotina

Subdivisión Deuteromycotina

Clase Deuteromycetes (=Hyphomycetes) 
Cuadro 1. Sistemas terrestres del estado de Colima.

\begin{tabular}{|c|c|c|c|c|}
\hline $\begin{array}{l}\text { Sistemas } \\
\text { terrestres }\end{array}$ & Localidades & Geomorfología & Clima & Vegetación \\
\hline Manantlán & $\begin{array}{l}\text { Zacualpan,Peña de Juluapan, Miscoate, } \\
\text { Los Sauces }\end{array}$ & Sierra & $\begin{array}{l}\text { Templado } \\
\text { húmedo } \\
\text { subhúmedo }\end{array}$ & $\begin{array}{l}\text { Encino, pino, } \\
\text { selva baja, media } \\
\text { y galería }\end{array}$ \\
\hline $\begin{array}{l}\text { Volcán } \\
\text { de Fuego }\end{array}$ & $\begin{array}{l}\text { San Antonio, Hierbabuena,Laguna María, } \\
\text { Parque Nacional }\end{array}$ & $\begin{array}{l}\text { Sierra- Eje } \\
\text { neovolcánico }\end{array}$ & $\begin{array}{l}\text { Templado } \\
\text { y cálido } \\
\text { húmedo }\end{array}$ & $\begin{array}{l}\text { Bosque pino, } \\
\text { encino, galería }\end{array}$ \\
\hline $\begin{array}{l}\text { Sierra } \\
\text { Compleja }\end{array}$ & $\begin{array}{l}\text { Las Cruces, Juárez, Tecusitán } \\
\text { Platanarillos, El Cobano, } \\
\text { Agustín Gómez, , El Charco }\end{array}$ & Sierra & $\begin{array}{l}\text { Cálido } \\
\text { subhúmedo }\end{array}$ & $\begin{array}{l}\text { Selva baja, } \\
\text { media, bosque } \\
\text { pino-encino }\end{array}$ \\
\hline $\begin{array}{l}\text { Valle } \\
\text { de Colima }\end{array}$ & $\begin{array}{l}\text { Barranca del muerto, La Trinidad, Los Limones, } \\
\text { El Chical, Villa de Alvarez, Comala, Minatitlán } \\
\text { Cuahutemoc }\end{array}$ & $\begin{array}{l}\text { Lomeríos } \\
\text { suaves y llanura }\end{array}$ & $\begin{array}{l}\text { Cálido } \\
\text { subhúmedo }\end{array}$ & $\begin{array}{l}\text { Selva baja, } \\
\text { Matorral }\end{array}$ \\
\hline $\begin{array}{l}\text { Valle del río } \\
\text { Armería }\end{array}$ & $\begin{array}{l}\text { La Limonera, El Chical, Armería, Tecomán, } \\
\text { Periqullos, } \\
\text { Magdalena, Los Amiales* }\end{array}$ & Valle Fluvial & Semi-seco & $\begin{array}{l}\text { Bosque de } \\
\text { Galería }\end{array}$ \\
\hline $\begin{array}{l}\text { Llanura } \\
\text { costera de } \\
\text { Tecomán }\end{array}$ & $\begin{array}{l}\text { El Naranjo, Boca de Apiza*, Tecomán*, El } \\
\text { Real*,El Paraíso* } \\
\text { Tecoanillo*,Boca de Pascuales* }\end{array}$ & $\begin{array}{l}\text { Llanura con } \\
\text { pendientes } \\
\text { suaves }\end{array}$ & $\begin{array}{l}\text { Cálido- } \\
\text { subhúmedo }\end{array}$ & $\begin{array}{l}\text { Selva baja, } \\
\text { matorral, manglar }\end{array}$ \\
\hline $\begin{array}{l}\text { Llanura } \\
\text { costera de } \\
\text { Cuyutlán }\end{array}$ & $\begin{array}{l}\text { Los Caracoles, Las Truchas, Santiago, Salahua, } \\
\text { Playa de Oro*, Cuyutlán* }\end{array}$ & Llanura Costera & $\begin{array}{l}\text { Cálido } \\
\text { subhúmedo } \\
\text { y semiseco }\end{array}$ & $\begin{array}{l}\text { Selva baja, } \\
\text { media, bosque } \\
\text { espinoso, } \\
\text { matorral y } \\
\text { manglar }\end{array}$ \\
\hline $\begin{array}{l}\text { Llanura costera } \\
\text { Potrero Grande }\end{array}$ & Colimilla, Isla Navidad, Cihuatlán, El Charco & Llanura Costera & $\begin{array}{l}\text { Cálido } \\
\text { subhúmedo }\end{array}$ & $\begin{array}{l}\text { Matorral, Selva } \\
\text { baja, manglar, } \\
\text { tular }\end{array}$ \\
\hline $\begin{array}{l}\text { Valle del río } \\
\text { Marabasco }\end{array}$ & Marabasco, Jalipa, Camotlán & $\begin{array}{l}\text { Valle } \\
\text { intermontano }\end{array}$ & $\begin{array}{l}\text { Cálido } \\
\text { húmedo y } \\
\text { subhúmedo }\end{array}$ & $\begin{array}{l}\text { Selva baja, } \\
\text { media, bosque de } \\
\text { galería }\end{array}$ \\
\hline $\begin{array}{l}\text { Sierra } \\
\text { Karstica }\end{array}$ & $\begin{array}{l}\text { Ixtlahuacán, Piscila, La Tunita, Callejones } \\
\text { La Trinidad }\end{array}$ & $\begin{array}{l}\text { Sierra con } \\
\text { cañadas }\end{array}$ & $\begin{array}{l}\text { Cálido } \\
\text { subhúmedo } \\
\text { y semiseco }\end{array}$ & $\begin{array}{l}\text { Selva baja y } \\
\text { media }\end{array}$ \\
\hline $\begin{array}{l}\text { Lomeríos } \\
\text { La Alzada }\end{array}$ & El Hervidero, Agua Caliente, Las Conchas & $\begin{array}{l}\text { Lomeríos y } \\
\text { llanura }\end{array}$ & $\begin{array}{l}\text { Cálido } \\
\text { subhúmedo }\end{array}$ & $\begin{array}{l}\text { Selva baja, } \\
\text { media, bosque de } \\
\text { galería }\end{array}$ \\
\hline
\end{tabular}

* Aislamiento del entomopatógeno. Hospedante. Hospedero y año de colecta se presentan en el Cuadro 2.

Orden Moniliales

Familia Moniliaceae

Género Hirsutella

Especie H. thompsonii

Variedades thompsonii, vinacea y sinemata

Una vez identificado como $H$. thompsonii, cada aislamiento se cultivó por dos días en medio líquido MEA, y después sobre una placa con una capa delgada $\left(5 \mathrm{~mm}^{2}\right)$ de MEA. Bajo condiciones estériles (campana de flujo), el micelio fúngico se salpicaó y distribuyó sobre toda la placa; posteriormente, con la punta opuesta de una pipeta Pasteur estéril, se hicieron oradaciones en medio de la placa, y los círculos del medio inoculado con el hongo se 


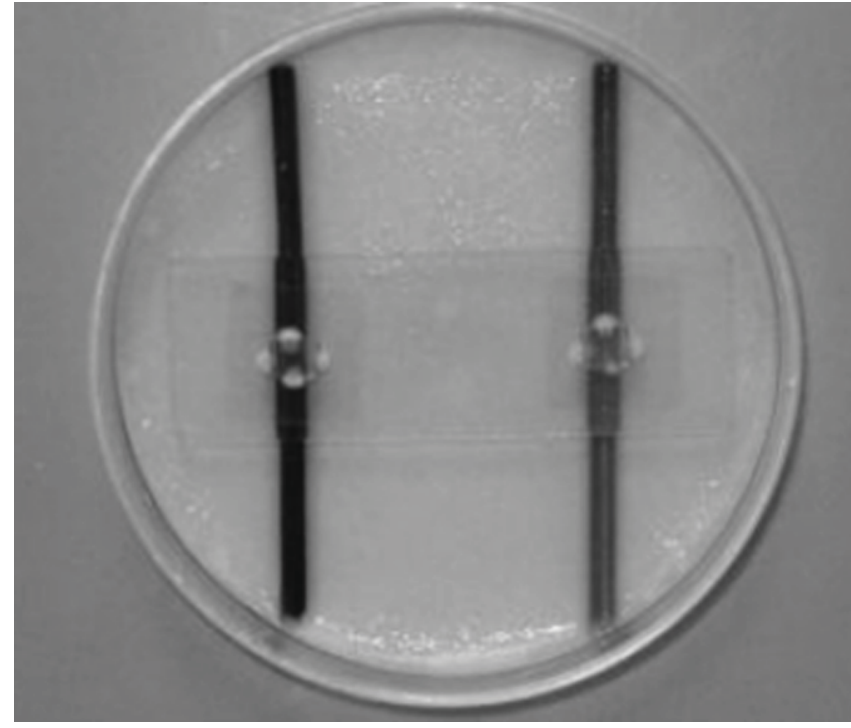

Figura 1. Técnica del microcultivo. Hirsutella thompsonii cultivado en una capa delgada de MEA sobre un cubreobjetos de vidrio.

transfirieron a portaobjetos estériles de cristal y se les colocó transfirieron a portaobjetos estériles de cristal y se les colocó un cubreobjetos. Las laminillas se pusieron dentro de cajas Petri de $9 \mathrm{~cm}$ de diámetro con filtro de papel húmedo y se incubaron a $27 \pm^{\circ} \mathrm{C}$ durante 9 días (Fig. 1). El micelio adherido a los cubreobjetos se tiñó usando lactofenol con $0.1 \%$ de azul de metileno para su observación en el microscopio óptico 340-400X. La morfología externa de los conidios, fiálides y micelio fue observada y se registró para cada uno de los aislamientos la distancia entre fiálides $(\mu \mathrm{m})$ y el diámetro de la colonia $(\mathrm{cm})$ (Lacey, 1997).

\section{Resultados}

Se aislaron 10 cepas de $H$. thompsonii; 5 de la variedad thompsonii y 5 de la variedad sinematosa; 6 sobre araña roja (T. urticae) (Fig. 2), 3 sobre eriófidos (A. guerreronis y P. oleivora) y 1 sobre brevipalpidos (Brevipalpus phoenicis) (Cuadro 2), en 3 sistemas terrestres de los 11 en los que se divide el estado de Colima y que presentan áreas citrícolas: Valle del río Armería (paisaje terrestre Coquimatlán, localidad Los Amiales), Llanura Costera de Tecomán (paisaje terrestre Tecomán y Chupadero, localidades Tecomán, Tecuanillo, El Real, Boca de Pascuales, El Paraíso y Boca de Apiza) y Llanura Costera de Cuyutlán (paisaje Terrestre Cuyutlán y Manzanillo, localidades Cuyutlán y Playa de Oro) (Fig. 3). El clima en estos sistemas es cálido subhúmedo y semiseco (Cuadro 1). De las cepas aisladas, 9 provienen de cultivos citrícolas y una (HtM150) de vegetación aislada (no agroecosistema) (Cuadro 2).

En laboratorio, la apariencia de la colonia fúngica, su crecimiento y la producción de conidios de las cepas aisladas
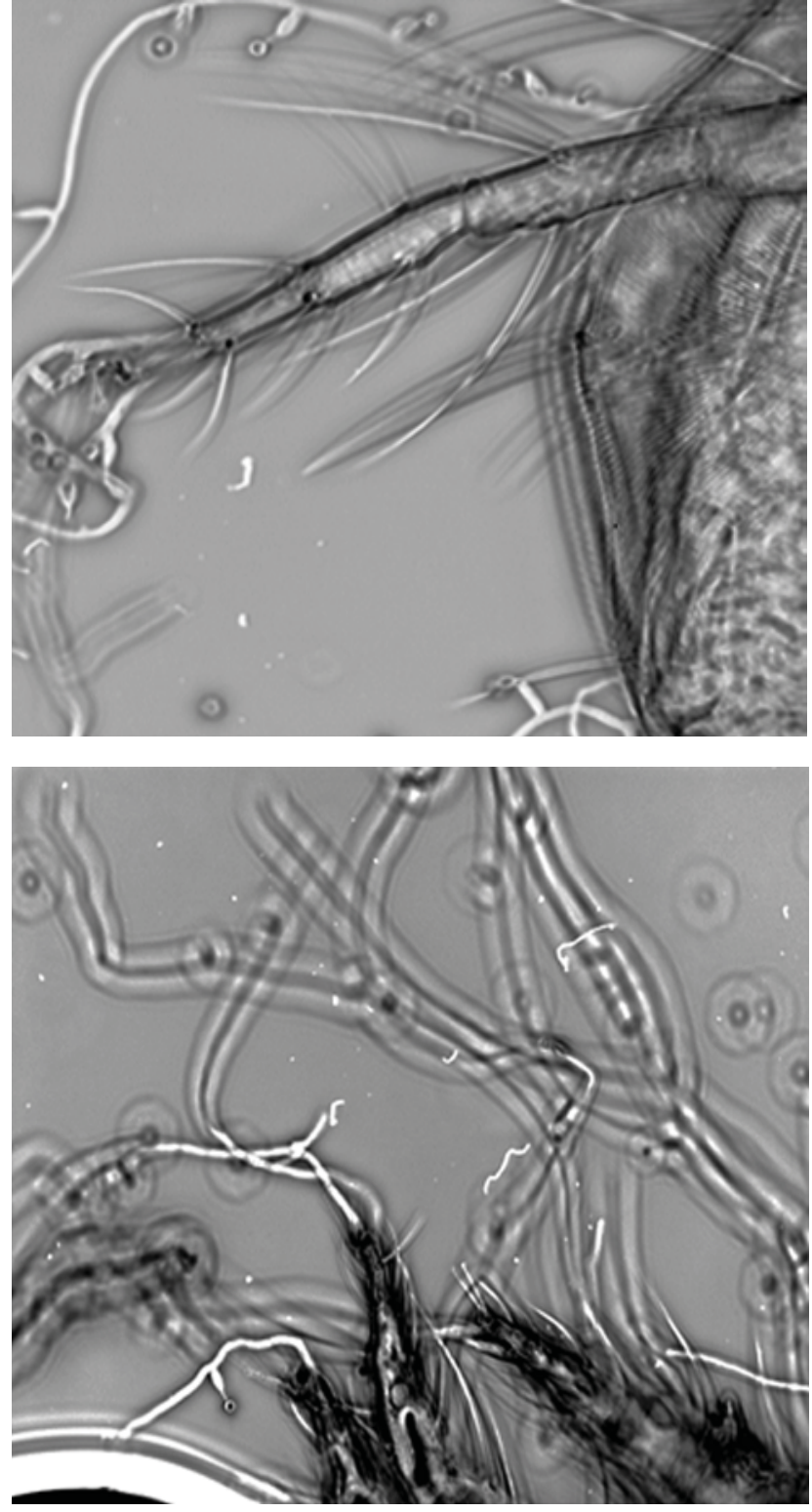

Figura 2. Presencia natural de $H$. thompsonii parasitando T. urticae (540X).

en Colima, presentaron diferencias. La morfología de la colonia se pudo categorizar por el color y elevación de la colonia (Cuadro 3). Algunas fueron delgadas, no elevadas y con poco crecimiento micelial aéreo, de coloración blanca a amarilla. El crecimiento aéreo del micelio con apariencia de polvo blanco, cerca de los márgenes de la colonia, principalmente en las de la variedad sinematosa. Los aislados de la variedad thompsonii, fueron ligeramente elevados, con mullido crecimiento micelial de color gris a gris verdoso, que es un rasgo característico de esta variedad (Fig.4). La distancia en micras entre fiálides de cada cepa aislada y el diámetro en centímetros de cada una de las colonias desarrolladas sobre las placas con MEA, después de 5 días de 
Cuadro 2. Relación del origen de los aislados de Hirsutella thompsonii de tres sistemas terrestres del estado de Colima, México.

\begin{tabular}{|c|c|c|c|c|c|c|}
\hline $\begin{array}{l}\text { Hirsutella } \\
\text { thompsonii } \\
\text { Clave en el } \\
\text { cepario }\end{array}$ & $\begin{array}{l}\text { Lugar de } \\
\text { colecta }\end{array}$ & $\begin{array}{l}\text { Sistema } \\
\text { terrestre }\end{array}$ & $\begin{array}{l}\text { Año de } \\
\text { colecta }\end{array}$ & Hospedante & Hospedero & Variedad \\
\hline HtM130 & $\begin{array}{l}\text { Boca de } \\
\text { Apiza }\end{array}$ & $\begin{array}{l}\text { LlanuraCostera } \\
\text { de Tecomán }\end{array}$ & 1999 & Tetranychus urticae & Cítricos & thompsonii \\
\hline HtM131 & Tecomán & $\begin{array}{l}\text { LlanuraCostera } \\
\text { de Tecomán }\end{array}$ & 1999 & Aceria guerreronis & Cocotero & thompsonii \\
\hline HtM135 & Tecuanillo & $\begin{array}{l}\text { LlanuraCostera } \\
\text { de Tecomán }\end{array}$ & 2000 & T. urticae & Cítricos & sinematosa \\
\hline HtM137 & El Real & $\begin{array}{l}\text { LlanuraCostera } \\
\text { de Tecomán }\end{array}$ & 2000 & T. urticae & Cítricos & sinematosa \\
\hline HtM138 & $\begin{array}{l}\text { Boca de } \\
\text { Pascuales }\end{array}$ & $\begin{array}{l}\text { LlanuraCostera } \\
\text { de Tecomán }\end{array}$ & 2000 & T. urticae & Cítricos & sinematosa \\
\hline HtM150 & Los Amiales & $\begin{array}{l}\text { Valle del río } \\
\text { Armería }\end{array}$ & 2002 & T. urticae & Guayabo & thompsonii \\
\hline HtM152 & El Paraíso & $\begin{array}{l}\text { LlanuraCostera } \\
\text { de Tecomán }\end{array}$ & 2002 & Brevipalpus phoenicis & Cítricos & sinematosa \\
\hline HtM155 & $\begin{array}{l}\text { Playa de } \\
\text { Oro }\end{array}$ & $\begin{array}{l}\text { LlanuraCostera } \\
\text { de Cuyutlán }\end{array}$ & 2003 & $\begin{array}{l}\text { Phyllocoptrupta } \\
\text { oleivora }\end{array}$ & Cítricos & thompsonii \\
\hline HtM160 & Tecomán & $\begin{array}{l}\text { LlanuraCostera } \\
\text { de Tecomán }\end{array}$ & 2003 & P. oleivora & Cítricos & thompsonii \\
\hline HtM164 & Cuyutlán & $\begin{array}{l}\text { LlanuraCostera } \\
\text { de Cuyutlán }\end{array}$ & 2004 & T. urticae & Cítricos & sinematosa \\
\hline
\end{tabular}

incubadas a $27-28^{\circ} \mathrm{C}$ se registran en el Cuadro 4 ; el $50 \%$ de los aislados en promedio midió de 1.56 a $1.86 \mathrm{~cm}$ de radio y 48.6 a $6.55 \mu \mathrm{m}$ de distancia entre fiálides; una cepa (HtM130) de la variedad thompsonii fue la que presentó menor diámetro en crecimiento radial; por otro lado, 2 aislados de la variedad sinematosa (HtM137 y HtM164) fueron los de mayor crecimiento radial y distancia entre fiálides.

\section{Discusión}

Los ácaros de las familias Eriophyidae y Tetranichidae se consideran plagas que causan daños de importancia económica a la agricultura en México y en el mundo; miembros de estos grupos atacan una amplia gama de plantas cultivadas (Dusso, 1993, Hill, 1994). Amrine y Stasny (1994) citan 2884 especies de eriófidos que viven sobre plantas, algunos de los cuales (376 especies) les inducen anormalidades, como la producción excesiva de pelos, formación de agallas (510 especies), producción de roña o decoloración (276 especies) y enrollamiento de las hojas (133 especies). También algunos eriófidos transmiten virus a las plantas, provocando pérdidas económicas mayores (Styer y Nault, 1996). La araña roja (T. urticae) se considera el tetraníquido de mayor importancia económica en el mundo (Ochoa et al., 1991; Young-Joon et al.,2001). En los sitios de muestreo (Cuadros 1 y 2) los tetraníquidos y los eriófidos fueron los grupos de ácaros con mayor abundancia; además, se encontraron parasitados algunos miembros de las familias Tenuipalpidae y Brevipalpidae.

En todo el mundo, los productos químicos son ampliamente utilizados para controlar las plagas en los cultivos; sin embargo, a mediano y largo plazo este método provoca resistencia en las plagas; además, en años recientes la gente comienza a poner más atención en su salud y en los riesgos a que se expone con esos productos, así como en el impacto que causan sobre el ambiente (Butt et al.,2001). El reconocimiento de otros métodos, como el biológico, son alternativas para minimizar el uso de químicos y mejorar la calidad del ambiente y de la salud. Muchos patógenos, 

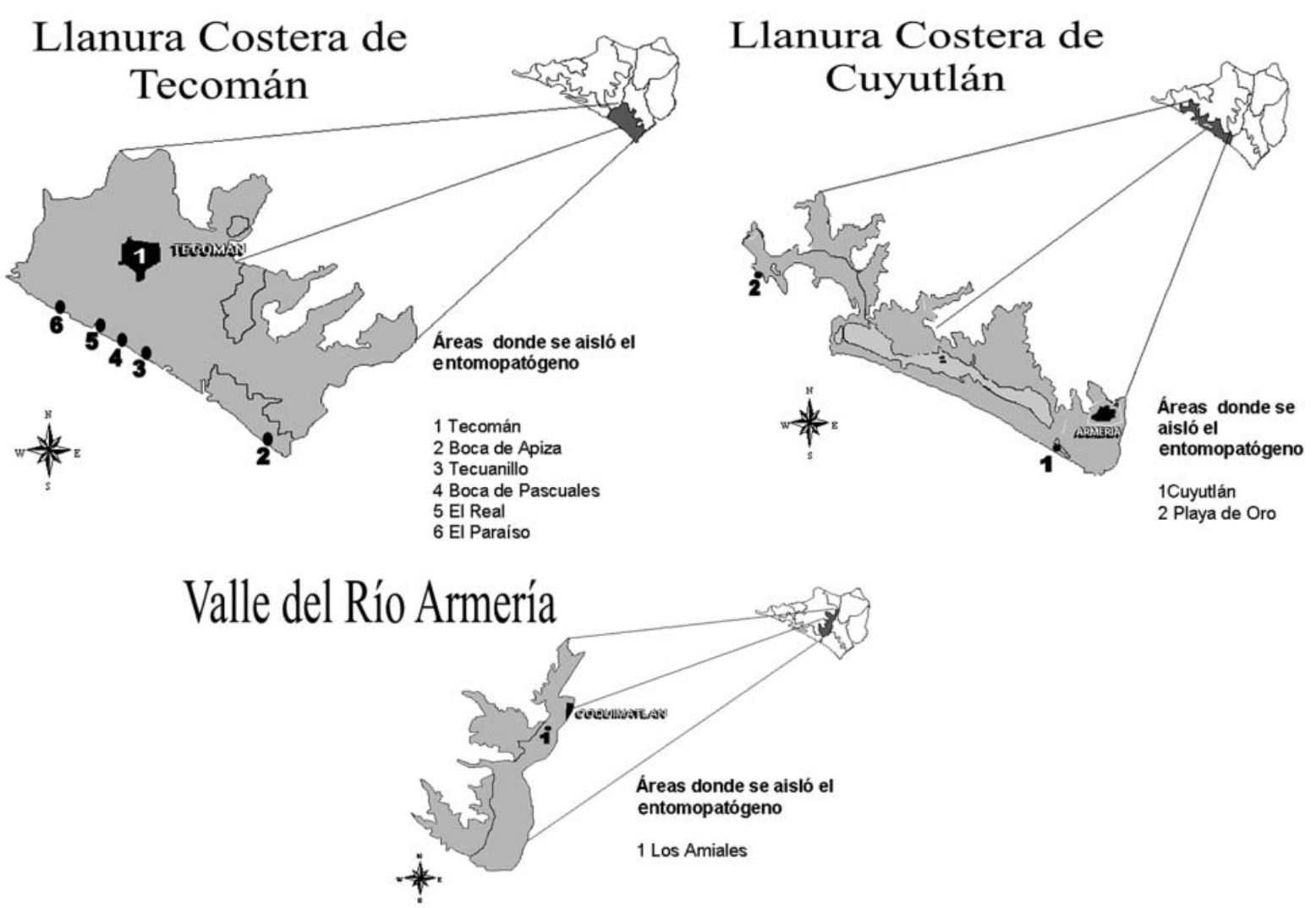

Figura 3. Localidades de los sistemas terrestres donde se aisló H. thompsonii.

Cuadro 3. Caracterización morfológica macroscópica de los aislados de $H$. thompsonii.

\begin{tabular}{|c|c|c|c|}
\hline $\begin{array}{l}\text { Cepa/ } \\
\text { variedad }\end{array}$ & Color & Elevación & Crecimiento \\
\hline $\begin{array}{l}\text { HtM135 } \\
\text { sinematosa }\end{array}$ & Blanco & Aplanada & Regular \\
\hline $\begin{array}{l}\text { HtM137 } \\
\text { sinematosa }\end{array}$ & $\begin{array}{l}\text { Blanco a } \\
\text { amarillo }\end{array}$ & Aplanada & Abundante \\
\hline $\begin{array}{l}\text { HtM138 } \\
\text { sinematosa }\end{array}$ & Blanco a gris & Afelpada & Abundante \\
\hline $\begin{array}{l}\text { HtM152 } \\
\text { sinematosa }\end{array}$ & Gris & $\begin{array}{l}\text { Afelpada- } \\
\text { aplanada }\end{array}$ & Regular \\
\hline $\begin{array}{l}\text { HtM164 } \\
\text { sinematosa }\end{array}$ & Blanco a gris & Afelpada & Abundante \\
\hline $\begin{array}{l}\text { HtM130 } \\
\text { thompsonii }\end{array}$ & Gris & Afelpada & Compacto \\
\hline $\begin{array}{l}\text { HtM131 } \\
\text { thompsonii }\end{array}$ & Blanco a gris & Afelpada & Abundante \\
\hline $\begin{array}{l}\text { HtM150 } \\
\text { thompsonii }\end{array}$ & $\begin{array}{l}\text { Gris a } \\
\text { amarillo }\end{array}$ & Aplanada & Compacto \\
\hline $\begin{array}{l}\text { HtM155 } \\
\text { thompsonii }\end{array}$ & Gris & $\begin{array}{l}\text { Afelpada- } \\
\text { aplanada }\end{array}$ & Abundante \\
\hline $\begin{array}{l}\text { HtM160 } \\
\text { thompsonii }\end{array}$ & Gris & Afelpada & Compacto \\
\hline
\end{tabular}

como bacterias, virus y hongos se producen comercialmente y se usan como agentes de control en varios países (Dent, 1995, Khetan, 2001 Vey et al., 2001; Khachatourians et al., 2002; Narayanan, 2002). De ahí la importancia de que en México se conozca la diversidad de enemigos naturales con que cuentan las plagas en los diferentes agroecosistemas y, así, utilizarlos como alternativa biológica, con mayor posibilidad de que se adapten a las condiciones ambientales de la región de donde son autóctonos y no tener la necesidad de importarlos de otros lugares.

El clima predominante de los sitios de colecta (Llanura Costera de Tecomán y Cuyutlán) de donde provienen todas las cepas aisladas, excepto la HtM50, es cálido subhúmedo, al igual que otros 7 sistemas terrestres (Cuadro 1) de los 11 que componen el estado de Colima (García, 1988). En el Valle del río Armería, el clima que predomina es el semiseco, pero las condiciones microclimáticas de la localidad (Los Amiales) donde se aisló la cepa HtM50 son más húmedas, por la cercanía de manantiales con abundante vegetación de selva baja y bosque de galería, lo que las asemeja a las condiciones de temperatura y húmedad predominantes en los agroecosistemas citrícolas, de donde provienen los ácaros infectados de los cuales se aisló el resto de las cepas que se citan en este trabajo. 

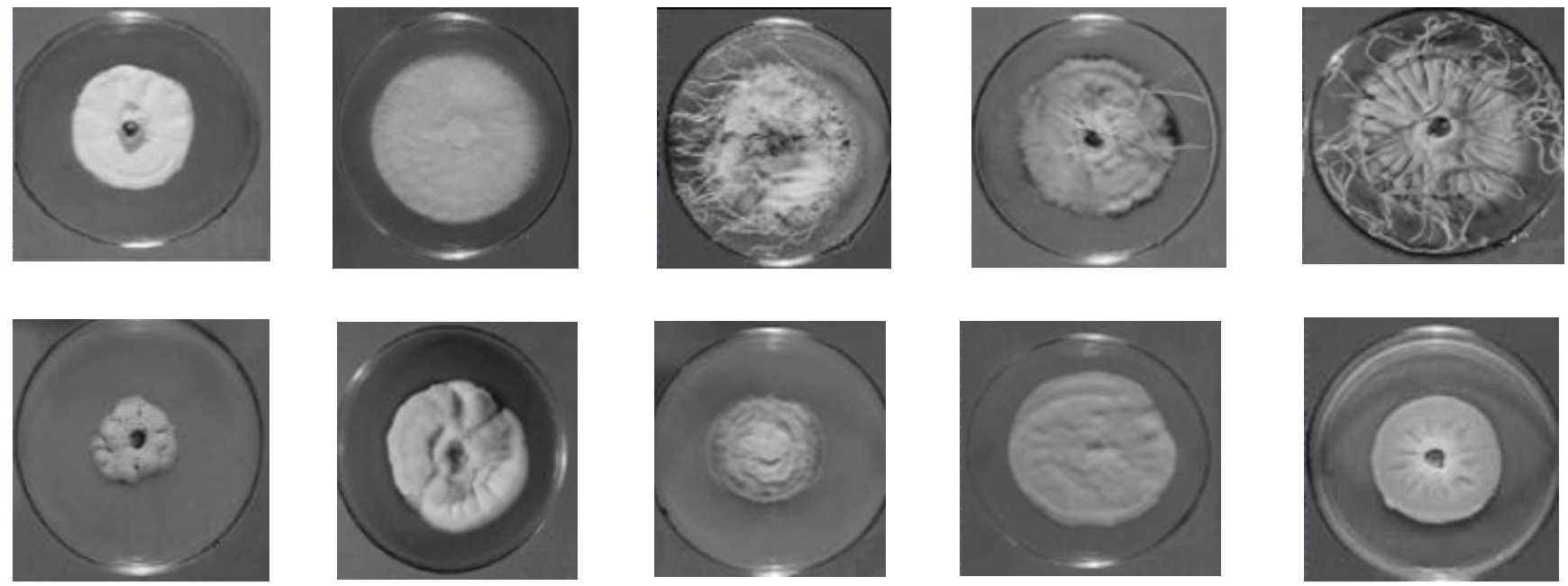

Figura 4. De izquierda a derecha, cepas de H. thompsonii. Parte superior HtM135, HtM137, HtM138, HtM152 y HtM164 (var. sinematosa). Parte inferior: HtM130, HtM131, HtM150 y HtM160 (var. thompsonii). Origen, hospedante, hospedero y fecha de colecta en los cuadros 1 y 2. Características morfológicas macroscópicas y microscópicas, cuadros 3 y 4.

Cuadro 4. Caracterización microscópica de las colonias. Crecimiento en placas con MEA después de 5 días de incubadas a $27-28^{\circ} \mathrm{C}$. Distancia entre fiálides $(\mu \mathrm{m})$ y diámetro $(\mathrm{cm})$.

\begin{tabular}{llllll}
\hline $\begin{array}{l}\text { Largo entre } \\
\text { fiálides }(\mu \mathrm{m})\end{array}$ & $\begin{array}{l}\text { Número } \\
\text { de } \\
\text { aislados }\end{array}$ & Cepas HtM: & $\begin{array}{l}\text { Diámetro de la } \\
\text { colonia }(\mathrm{cm})\end{array}$ & $\begin{array}{l}\text { Número } \\
\text { de aislados }\end{array}$ & Cepas HtM: \\
\hline $33.5-48.5$ & 1 & 130 & $1.12-1.55$ & 1 & 130 \\
$48.6-66.5$ & 5 & $131,135,150,152,160$ & $1.56-1.86$ & 4 & $131,135,150,160$ \\
$66.6-86.0$ & 2 & 138,155 & $1.87-2.13$ & 2 & 138,152 \\
$86-01-126.07$ & 2 & 137,164 & $2.14-3.94$ & 3 & $137,155,164$ \\
\hline
\end{tabular}

En las zonas templadas al norte y noreste del estado, se encontraron algunos ejemplares parasitados sobre vegetación nativa aislada (es decir, no cultivos), y en árboles de mango y guayabo. En este caso, los entomopatógenos fueron de crecimiento más lento que los encontrados en los agroecosistemas, por lo que el crecimiento de estos aislados fue enmascarado y/o inhibido por el de saprofitos de los ejemplares provenientes de la vegetación nativa del resto de los sistemas terrestres en que se divide Colima que finalmente impidieron el aislamiento de cepas puras.

El crecimiento más acelerado de las cepas aisladas en los agroecosistemas citrícolas se puede explicar de dos maneras; la primera, debido al efecto sinérgico que tienen sobre $H$. thompsonii algunos productos químicos a bajas concentraciones (Sosa-Gomez y Nasca, 1983; Bello et al., 1987), fenómeno que seguramente ocurre en los cultivos por la desnaturalización que ocasiona el ambiente a los ingredientes activos de los químicos que se aplican (aunque el uso excesivo y a altas concentraciones de éstos puede inhibirlos o matarlos); por otro lado, se puede explicar por la capacidad de adaptación del hongo en su supervivencia para eficientizar la interacción patógeno-hospedero bajo las condiciones de estrés y confort que los cultivos citrícolas les brindan (Jolivet, 1992; Thompson, 1994), que inducen a los hongos a desarrollar fiálides más elongadas o a esporular más rápidamente, generando mayor desarrollo micelial y capacidad infectiva para generar epizootias (Butt et al., 1995, Hajeck y St. Leger. 1994). Cabe señalar que un aspecto característico de $H$. thompsonii es el polimorfismo y las diferencias de comportamiento bajo diferentes condiciones para esporular (Rosas, et al., 1994; 1995), para expresar los metabolitos secundarios en su fase vegetativa (Mozes-Koch et al, 1995; Maimala et al.,2002; Rosas et al., 2003) y su patogenicidad (Vey et al., 1993; Maimala et al.,2002; Rosas et al., 2003); todo esto facilitó el éxito en el aislamiento, en comparación con las cepas que parasitan a hospedantes sobre hospederos de áreas no perturbadas que presentaron crecimiento muy lento e impidieron obtener aislados puros, por la competencia con otros microorganismos asociados al cuerpo de los ácaros, como ocurre con otros insectos (Lacey, 
1997; Sosa-Gómez et al., 1997).

El tipo de crecimiento y coloración de las cepas no se correlacionan con el sistema terrestre donde se encontraron ni con las condiciones climáticas imperantes en ellos. La cepa HtM130 de H. thompsonii var. thompsonii, presentó formación de exudados en la fase esporulativa, aunque en menor proporción que los producidos por la cepa HtM120I aislada en el estado de Guerrero (Rosas et al., 2003). Estos metabolitos fueron caracterizados no tóxicos para ácaros, pero inhiben la oviposición en las hembras de T. urticae durante una semana (Rosas et al., 2003; 2004), fenómeno que interrumpe el ciclo reproductivo de la plaga, disminuyendo el número de ácaros por planta y el efecto de las poblaciones de ácaros sobre el follaje y frutos. Si tomamos en consideración que bajo condiciones óptimas el ciclo biológico de esta plaga es de dos semanas (Tsagkarakou et al., 1996), la cepa colimota HtM130 y las guerrerenses HtM5 y HtM120I tienen mayor potencial para incluirse en estrategias de manejo para el control de tetraníquidos en cítricos, que el resto de los aislados de la zona.

La distancia entre fiálides y el tamaño de la colonia presentó leve diferencia en las cepas HtM152 (H. thompsonii var. sinematosa) y HtM155 (H. thompsonii var. thompsonii), en relación con el resto de los aislados, lo que demuestra una vez más la naturaleza pleomórfica de este hongo (Boucias y Pendland, 1998); éstos aspectos y la capacidad de esporulación se relacionan con el grado de patogenicidad y agresividad del hongo (Rosas et al.,1995) y puede corresponder, o no, a la expresión del gen de la toxina hirsutelina (HtA) (Maimala et al., 2002) y a la expresión de los metabolitos inhibidores de la oviposición (Rosas et al.,2003). De ahí la importancia de contar con aislamientos autóctonos para su uso y garantizar su mejor establecimiento bajo las condiciones agroclimáticas de la región donde se pretenda utilizarlas

Es evidente que la agricultura moderna en el estado de Colima ha aportado beneficios a la población mediante el incremento significativo de la producción y la calidad de la mayoría de los cultivos agrícolas; sin embargo, este beneficio se pone en duda debido a los numerosos efectos negativos ocasionados al ambiente y a la salud humana, además de inducir a la pérdida de la biodiversidad. Es necesario conocer los enemigos naturales de las plagas de importancia agrícola, para implantar estrategias para el establecimiento de todas aquellas prácticas que puedan reducir los problemas de contaminación ambiental y salud sin afectar la producción de alimentos. La presente contribución sobre la diversidad de este entomopatógeno ( $H$. thompsonii) en una región agrocitrícola muestra que antes de introducir enemigos comerciales o aislamientos de otros sitios es importante valorar la biodiversidad de los enemigos naturales presentes en las condiciones locales (en este caso hongos), para no afectar los sistemas naturales de regulación.

A la fecha el laboratorio de Control Biológico y Biotecnología de la UCDR cuenta con una extensa colección de aislamientos de $H$. thompsonii (121) de varias partes del país y del extranjero, con potencial para diferentes especies de ácaros, como A. guerreronis, T. urticae, Brevipalpus spp., P.oleivora, Varroa sp, etc., a los cuales se agregan las 10 cepas de la diversidad biológica del estado de Colima, México, que aquí se registran.

\section{Literatura citada}

Amrine, J. W. Jr. y T. A. H. Stasny. 1994. Catalog of the Eriophyoidea (Acarina: Prostigmata) of the World. Indira, West Bloomfield, Michigan. 804 p.

Bello, J. L., J. L. Rosas-Acevedo, L. Sanpedro y C. Toriello. 1987. Efecto de cuatro pesticidas en el crecimiento de ocho cepas de Hirsutella thompsonii (Fisher). In Resúmenes del XVIII Congreso Nacional de Microbiología, Acapulco, Guerrero. p. 77.

Boucias, D. G y J. C. Pendland. 1998. Principles of insect pathology. Kluwer Academic, Norwell, Massachusetts. $537 \mathrm{p}$.

Butt, T. M., C. Jackson y N. Magan. 2001. Introduction - Fungal biological control agents: progress, problems and potential. In Fungi as biocontrol agents progress, problems and potential, T. M. Butt, C. Jackson y N. Magan (eds.). CABI, Wallingford, Oxon. 1-8 p.

Butt, T. M., L. Ibrahim, S. J. Clark y A. Beckett. 1995. Germination behaviour of Metarhizium anisopliae on the surface of aphid and flea beetle cuticles. Mycological Research 99: 945-950.

Cabrera, R. I. y D. Domínguez. 1987. Estudio de dos especies de Hirsutella y sus hospedantes en el cultivo de la guayaba Psidium guajava. Agrotecnia de Cuba 19(1): 29-34.

Chandler, D., G. Davidson, J. K. Pell, B.V. Ball, K. Shaw y K. D. Sunderland. 2000. Fungal biocontrol of acari. Biocontrol Science and Technology 10: 357-384.

Dirección de Ecología de Colima. 1993. Programa de ordenamiento ecológico del territorio del estado de Colima. Gobierno de Estado de Colima, Secretaría de Desarrollo Urbano, Dirección de Ecología, UNESCOCENEDIC, Universidad de Colima.

Dent, D.R. 1995. Control measures, Chapter 3. In Integrated pest management, D. R. Dent (ed.). Chapman \& Hall, London. p. 47-85.

Duso, C. 1993. Factors affecting the potential of phytoseiid mites (Acari: Phytoseiidae) as biocontrol agents in NorthItalian vineyards. Experimental and Applied Acarology 17: 241-258.

Fisher, F. E., J. T. Griffiths y L. Thompson. 1949. An epizootic of Phyllocoptruta oleivora (Ashm.) on citrus in Florida. Phytopathology 39: 510-512.

García, E. 1987. Modificaciones al sistema de clasificación climática de Köppen. Offset Larios, México. 217 p.

Hajeck, A. E. y R. J. St. Leger. 1994. Interactions between 
fungal pathogens and insects hosts. Annual Review of Entomology 39: 293-322.

Hill, D. S. 1994. Agricultural Entomology. Timber Press, Portland.

Hodge, K. T., N. M. Viaene y W. Gams. 1997. Two Harposporium species with Hirsutella synanamorphs. Mycology Research 101(11): 1377-1382.

Jolivet, P. 1992. Insects and plants: parallel evolution and adaptations, $2^{\text {nd }}$ edition. Sandhill Crane, Gainesville, Florida. p. 157-163.

Khachatourians, G. G., E. P. Valencia y G. S. Miranpuri. 2002. Beauveria bassiana and entomopathogenic fungi in the management of insect pests. In Microbial biopesticides, O. Koul and G. S. Dhaliwal (eds.). Taylor \& Francis, New York. p. 239-275.

Khetan, S. K. 2001. Microbial pest control. Marcel Dekker, New York. 300 p.

Lacey, L. A. 1997. Manual of techniques in insect pathology. Biological Techniques. Academic, San Diego, California.

Mazet, I. y A. Vey. 1995. Hirsutellin A, a toxic protein produced in vitro by Hirsutella thompsonii. Microbiology 141: 1343-1348.

McCoy, C. W. 1996. Pathogens of Eriophids mites. In Eriophyoid mites their biology, natural enemies and control, E. E. Linquist, M. W. Sabelis y J. Bruin (eds.). Elsevier, Amsterdam, p. 481-490.

McCoy, C. W., y R. F. Kanavel. 1969. Isolation of Hirsutella thompsonii from the citrus rust mite, Phyllocoptruta oleivora and its cultivation on various synthetic media. Journal of Invertebrate Pathology 14: 386-390.

McCoy, C. W., R. A. Samsom y D. G. Boucias. 1988. In Handbook of pesticides, vol.V. Microbial insecticides. Part. A. Entomogenous protozoa and fungi, C. M. Ignoffo (ed.). CRC, Boca Ratón, Florida, p. 151-243.

Maimala, S., A. Tartar, D. G. Boucias, y A. Chandrapatya. 2002. Detection of the toxin Hirsutellin A from Hirsutella thompsonii. Journal of Invertebrate Pathology 80: 112-126.

Mains, E. B. 1951. Entomogenous species of Hirsutella, Tilachlidium and Synnematium. Mycologia 43: 691-717.

Minter, D. W. y B. L. Brady. 1980. Mononematous species of Hirsutella. Transations British Mycology Society 74: 271-282.

Mozes-Koch, R., O. Edelbaum, O. Livneh, O. Sztejnberg, A. Uziel, U. Gerson y I. Sela. 1995. Identification of Hirsutella species, isolates within a species and intraespecific heterokaryons by random amplified polymorphic DNA (RAPD). Journal of Plant Dieseases and Protection 102: 284-290.

Muma, M. H. 1955. Factors contributing to the natural control of citrus insects and mites in Florida. Journal of Economic Entomology 48: 432-438.

Narayanan, K. 2002. Microbial control of insect pests: role of genetic engineering and tissue culture. In Microbial Biopesticides, O. Koul and G. S. Dhaliwal (eds.). Taylor
\& Francis, New York, p. 248-276.

Navajas, M., J. Lagnel, J. Gutiérrez y P. Boursot. 1998. Species-wide homogeneity of nuclear ribosomal ITS2 sequences in the spider mite Tetranychus urticae contrasts with extensive mitochondrial COI polymorphism. Heredity 80: 742-752.

Ochoa, R., H. Aguilar y C. Vargas. 1991. Ácaros fitófagos de América Central: guía ilustrada. Centro Agronómico Tropical de Investigación y Enseñanza (CATIE), Turrialba. $251 \mathrm{p}$.

Roberts, D. W. 1989. World picture of biological control of insects by fungi. Memorias Instituto Oswaldo Cruz. Río de Janeiro 34 (Suplemento III): 89-100.

Roberts, D. W. y A. S. Campbell. 1977. Stability of entomopathogenic fungi. Miscelaneous Publisher Entomology of Society of America 10: 1-25.

Rombach, M. C. y A. J. van Winkelhoff. 1981. Contributions to the taxonomy of the genus Hirsutella. Preliminary classification based on host-fungus relation. Centralbureau Voor Schimelcultures, Baarn. 1-23 p.

Rosas-Acevedo, J. L., R. Alatorre, J. Valdez y L. Sampedro. 1994. Crecimiento micelial y esporulación de siete cepas de Hirsutella thompsonii y una de $H$. nodulosa en seis medios líquidos. Agrociencia 5: 91-101.

Rosas-Acevedo, J. L, R. Alatorre, L. Sampedro y J. Valdez. 1995. Esporulación de los hongos entomopatógenos Hirsutella thompsonii. Fisher y $H$. nodulosa Petch en cultivo mixto. Revista Latinoamericana de Microbiología 37: $59-64$.

Rosas-Acevedo, J. L., D. G. Boucias., R. Lezama, K. Sims y A. Pescador. 2003. Exudate from sporulating cultures of Hirsutella thompsonii, inhibit oviposition by the twospotted spider mites Tetranychus urticae. Applied and Experimental Acarology 39: 213-225.

Rosas-Acevedo, J. L., D. G. Boucias, R. Lezama, O. Rebolledo, A. Y. Rosas-Acevedo, A. Sánchez-Infante, W. Mejía y L. Sampedro. 2004. La araña roja Tetranychus urticae Koch (Acarina:Tetranychidae) afectada por Hirsutella thompsonii Fisher. Entomología Mexicana 3: 354-357.

Rosas-Acevedo, J. L. y L. Sampedro. 1991. Parasitismo de Hirsutella thompsonii Fisher sobre Phyllocoptruta oleivora (Ashm.) y Aceria guerreronis Keifer. Boletín de la Sociedad Mexicana de Entomología 9: 42-45.

Rosas-Acevedo, J. L. y L. Sampedro. 1992. Presencia del entomopatógeno Hirsutella thompsonii Fisher en el estado de Guerrero. Ciencia 6: 18-25.

Samsom, R. A., C. W. McCoy y K. L. O’Donnell. 1980. Taxonomy of the acarine parasite Hirsutella thompsonii. Mycologia 72: 359-377.

Spears, A. T. y W. W. Yothers. 1924. Is there an entomogenous fungus attacking the citrus rust mite in Florida ? Science 60: 41-42.

Sosa-Gómez. D. F., D. G. Boucias, y J. L. Nation. 1997. 
Attachment of Metarhizium anisopliae to the southern green stink bug Nezara viridula cuticle and fungistatic effect of cuticular lipids and aldehydes. Journal of Invertebrate Pathology 69: 31-39.

Sosa-Gómez. D. F. y A. J. Nasca. 1983. Primera cita del hongo patógeno de ácaros Hirsutella thompsonii (Fisher, 1950) para la República Argentina. CIRPON Revista de Investigación 1: 137-141.

Styer, W. E. y L. R. Nault. 1996. Damage and control of eriophyoid mites in crops: corn and grain plants. In Eriophyoid mites: their biology, natural enemies and control, E. E. Lindquist, M. W. Sabelis and J. Bruin (eds.). Elsevier,Amsterdam, p.611-618.

Tsagkarakou, A., M. Navajas, J. Lagnel, J. Gutiérrez y N. Pasteur. 1996. Genetic Variability in Tetranychus urticae (Acari:Tetranychidae) from Greece: insecticide resistance and isozymes. Journal of Economic Entomology 89: 1354-1358.

Thompson, J. 1994. The coevolutionary process. University of Chicago Press, Chicago, Illinois. 383 p.

Van der Geest, L. P. S., S. L. Elliot, J. A. J. Breeuwer y E. A.
M. Beerling. 2000. Disease of mites. Experimental and Applied Acarology 24: 497-560.

Vey, A., J. M. Quiot, I. Mazet y C. W. McCoy. 1993. Toxicity and pathology of crude broth filtrate produced by Hirsutella thompsonii var. thompsonii in shake culture. Journal of Invertebrate Pathology 61: 131-137.

Vey, A., R. E. Hoagland and T. M. Butt. 2001. Toxic metabolites of fungal biocontrol agents. In Fungi as biocontrol agents progress, problems and potential, T. M.

Butt, C. Jackson and N. Magan (eds.).CABI, Wallingford, Oxon. p. 311-346.

Wilson, L. J., G. A. Herron, L. R. Bauer y D. A. Lally. 1999. Acaricidal and stimulatory effects on insecticides on Tetranychus urticae Koch (Acari:Tetranychidae) in cotton. Australian Journal of Entomology 38: 30-33.

Young-Joon, A., K. Young-Joon y Y. Jai-Ki. 2001. Toxicity of the herbicide glufosinate-ammonium to predatory insects and mites of Tetranychus urticae (Acari: Tetranychidae) under laboratory conditions. Journal of Economic Entomololgy 94: 157-161. 